\title{
Observational Study Designs: Synopsis for Selecting an Appropriate Study Design
}

\author{
Assad A. Rezigalla ${ }^{1}$ \\ 1. Department of Basic Medical Sciences, College of Medicine, University of Bisha, Bisha, SAU
}

Corresponding author: Assad A. Rezigalla, arezigalla@ub.edu.sa

\begin{abstract}
The selection of a study design is the most critical step in the research methodology. Crucial factors should be considered during the selection of the study design, which is the formulated research question, as well as the method of participant selection. Different study designs can be applied to the same research question(s). Research designs are classified as qualitative, quantitative, and mixed design. Observational design occupies the middle and lower parts of the hierarchy of evidence-based pyramid. The observational design is subdivided into descriptive, including cross-sectional, case report or case series, and correlational, and analytic which includes cross-section, case-control, and cohort studies. Each research design has its uses and points of strength and limitations. The aim of this article to provide a simplified approach for the selection of descriptive study design.
\end{abstract}

Received 11/19/2019

Review began 11/25/2019 Review ended 12/15/2019 Published 01/17/2020

() Copyright 2020

Rezigalla. This is an open access article distributed under the terms of the Creative Commons Attribution License CC-BY 3.0., which permits unrestricted use, distribution, and reproduction in any medium, provided the original author and source are credited.
Categories: Medical Education, Miscellaneous, Epidemiology/Public Health

Keywords: observational, study design, descriptive, retrospective, prospective, strength

\section{Introduction And Background}

A research design is defined as the "set up to decide on, among other issues, how to collect further data, analyze and interpret them, and finally, to provide an answer to the question" [1]. The primary objective of a research design is to guarantee that the collected evidence allows the answering of the initial question(s) as clearly as possible [2]. Various study designs have been described in the literature [1-3]. Each of them deals with the specific type of research or research questions and has points of strength and weakness. Broadly, research designs are classified into qualitative and quantitative research and mixed methods [3]. The quantitative study design is subdivided into descriptive versus analytical study designs or as observational versus interventional (Figure 1). Descriptive designs occupy the middle and lower parts of the hierarchy of evidence-based medicine pyramid. Study designs are organized in a hierarchy beginning from the basic "case report" to the highly valued "randomised clinical trial" [4-5].

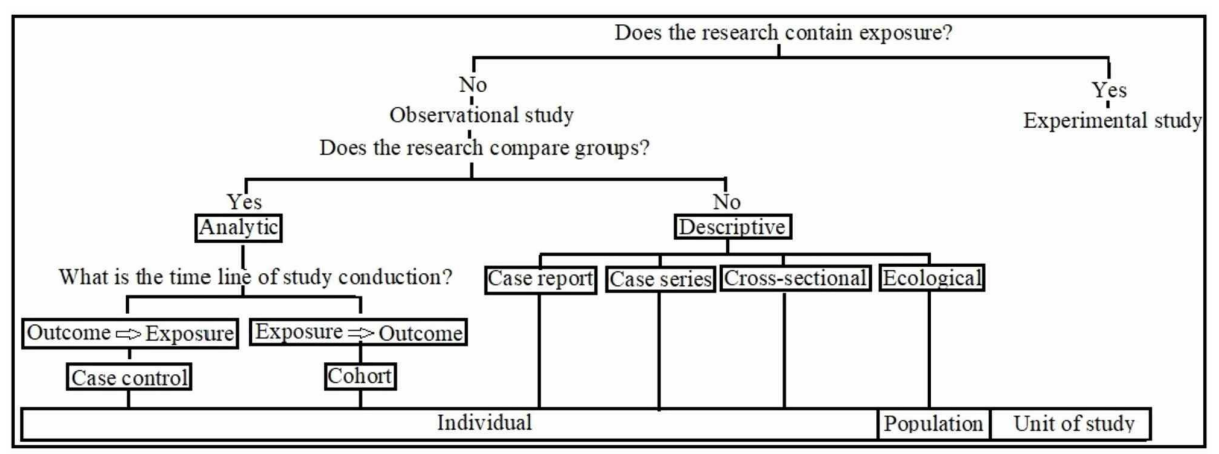

FIGURE 1: Classification of observational study design.

\section{Review}

\section{Case report}

The case report describes an individual case or cases in their natural settings. Also, it describes unrecognized syndromes or variants, abnormal findings or outcomes, or association between risk factors and disease. It is the lowest level and the first line of evidence and usually deals with the newly emerging issues and ideas (Table 1) [4, 6-10]. 


\section{Cureus}

\section{Case Report Design}

Strengths $[4,6-9]$

Identification of new, abnormal, or variant presentation of diseases.

Have significant educational value.

Help in generating a hypothesis.

Researching rare or uncommon disorders.

In-depth narrative case studies.

Flexible structure.
Limitations $[6,9]$

Lack of generalizability and implications.

Uncontrolled.

Selection bias.

No epidemiological indices (parameters).

Over-interpretation.

Confidentiality.

Causes may have other explanations.

TABLE 1: Strengths and Limitations of Case Report Design

\section{Case series}

A case series is a report on data from a subject group (multiple patients) without control $[6,11$ -

12]. Commonly, this design is used for the illustration of novel, unusual, or atypical features identified in medical practice [6]. The investigator is governed by the availability and accuracy of the records, which can cause biases [13-14]. Bias in a case series can be decreased through consecutive patient enrollment and predefined inclusion and exclusion criteria, explicit specification of study duration, and enrollment of participants (Table 2) [11-12].

\section{Case Series}

Strengths $[6,11-12]$

Educational.

It described the outcomes of novel treatments.

The gained information can be used to generate hypotheses.

Provide strong evidence with multiple cases.

Helpful in refining new techniques or treatment protocols.

Identify the rare manifestations of a disease or drug.
Limitations [6, 11-12]

Selection bias.

Lack of control.

Difficult to compare different cases.

The result may not be generalized.

Immediate follow-up.

Have a lower position on the hierarchy of evidence.

Feasible study designs.

\section{TABLE 2: Strengths and Limitations of Case Series Design}

\section{Correlational study design}

Correlational studies (ecologic studies) explore the statistical relationships between the outcome of interest in population and estimate the exposures. It deals with the community rather than in individual cases. The correlational study design can compare two or more relevant variables and reports the association between them without controlling the variables. The aim of correlational study design or research is to uncover any types of systematic relationships between the studied variables. Ecological studies are often used to measure the prevalence and incidence of disease, mainly when the disease is rare. The populations compared can be defined in several ways, such as geographical, time trends, migrants, longitudinal, occupation, and social class. It should be considered that in ecological studies, the results are presented at the population (group) level rather than individuals. Ecological studies do not provide information about the degree or extent of exposure or outcome of interest for particular individuals within the study group (Table 3) [7, 15-16]. For example, we do not know whether those individuals who died in the study group under observation had higher exposure than those remained alive. 


\section{Cureus}

\section{Correlational study design}

Strengths $[15-16]$

Quick and easy.

Describes the strength of relationships.

It is used to assess behavior.

Predictor variables cannot be manipulated.

Uses of data records.

\section{Limitations [15-16]}

Correlations do not equal causation.

Correlations can be misused.

Cannot be used to identify causal relationships

It cannot provide certain information.

TABLE 3: Strengths and Limitations of Correlation Study Design

\section{Cross-sectional study design}

The cross-sectional study examines the association between exposures and outcomes on a snap of time. The assessed associations are guided by sound hypotheses and seen as hypothesis-generating [17]. This design can be descriptive (when dealing with prevalence or survey) or analytic (when comparing groups) [17-18]. The selection of participants in a cross-sectional study design depends on the predefined inclusion and exclusion criteria [18-19]. This method of selection limits randomization (Table 4).

Cross-sectional Study Design

Strengths of $[17,19-20]$

Fast and inexpensive.

Useful for planning monitoring and evaluation of public health.

Efficient in studying rare diseases.

There are seldom ethical difficulties.

It can assess multiple outcomes.

Population-based surveys.

Estimation of prevalence.

Calculation of odds ratio.

The baseline for a cohort study.
Limitations [17, 19-20]

Difficult to derive causal relationships.

Prone to certain types of biases.

The response rate is critical.

The temporality of the design.

No clear demarcation between exposure and effect.

\section{TABLE 4: Strengths and Limitations of Cross-sectional Study Design}

\section{Case-control study}

A case-control study is an observational analytic retrospective study design [12]. It starts with the outcome of interest (referred to as cases) and looks back in time for exposures that likely caused the outcome of interest $[13,20]$. This design compares two groups of participants - those with the outcome of interest and the matched control [12]. The controls should match the group of interest in most of the aspects, except for the outcome of interest [18]. The controls should be selected from the same localization or setting of the cases [13, 21-22]. Case-control studies can determine the relative importance of a predictor variable about the presence or absence of the disease (Table 5). 


\section{Cureus}

Case-control Study Design

Strengths [12, 20-21]

Limitations [12, 20-21]

Relatively fast in conduction in comparison with prospective cohort studies.

Not useful for rare exposures.

Comparatively, needs few participants and fewer resources.

Cannot estimate the incidence.

Useful for testing hypotheses.

Affect by observation and recall bias.

Useful in studying multiple exposures in the same outcome.

Can study the association of risk factors and outcomes in outbreak investigations.

It can generate much information from relatively few participants with unusual cases.

Feasible in diseases with a long latent period.

TABLE 5: Strengths and Limitations of Case-control Study Design

\section{Cohort study design}

The cohort study design is classified as an observational analytic study design. This design compares two groups, with exposure of interest and control one [12, 18, 22-24].

Cohort design starts with exposure of interest comparing them to non-exposed participants at the time of study initiation [18, 22, 24]. The non-exposed serve as external control. A cohort design can be either prospective [18] or retrospective [12, 20,24-25]. In prospective cohort studies, the investigator measures a variety of variables that might be a risk factor or relevant to the development of the outcome of interest. Over time, the participants are observed to detect whether they develop the outcome of interest or not. In this case, the participants who do not develop the outcome of interest can act as internal controls. Retrospective cohort studies use data records that were documented for other purposes. The study duration may vary according to the commencement of data recording. Completion of the study is limited to the analysis of the data [18, 22, 24]. In 2016, Setia reported that, in some instances, cohort design could not be well-defined as prospective or retrospective; this happened when retrospective and prospective data were collected from the same participants (Table 6) [24].

\section{Cohort Study Design}

Strengths $[12,20,24]$

The temporality between exposure and outcome is well-defined.

Study multiple outcomes in the same exposure.

Efficient in rare outcomes if the rare outcome is common in some exposures.

Accurate measure of variables in prospective cohort design.

The retrospective cohort is relatively fast in conduction and inexpensive.

Lack of bias in the retrospective cohort because the collected data was not initially for research.

It can measure potential causes and relative risk.

\section{Limitations $[12,20,24]$}

Inability to control all the confounding variables.

A prospective cohort design is time-consuming and costly.

Variables in the retrospective cohort study may not be very accurate since the collected data was not intended for research purposes.

May not be very useful in case of rare outcomes.

In the prospective cohort design, the loss of follow-up is a critical problem.

Retrospective cohorts may be affected by recall bias.

Ethical problems

TABLE 6: Strengths and Limitations of Cohort Study Design

The selection of the study design is the most critical step in research methodology [4, 26]. An appropriate study design guarantees the achievement of the research objectives. The crucial factors that should be considered in the selection of the study design are the formulated research question, as well as the method 
of sampling $[4,27]$. The study design determines the way of sampling and data analysis [4]. The selection of a research study design depends on many factors. Two crucial points that should be noted during the process selection include different study designs that may be applicable for the same research question(s) and researches may have grey areas in which they have different views about the type of study design [4].

\section{Conclusions}

The selection of appropriate study designs for research is critical. Many research designs can apply to the same research. Appropriate selection guarantees that the author will achieve the research objectives and address the research questions.

\section{Additional Information \\ Disclosures}

Conflicts of interest: In compliance with the ICMJE uniform disclosure form, all authors declare the following: Payment/services info: All authors have declared that no financial support was received from any organization for the submitted work. Financial relationships: All authors have declared that they have no financial relationships at present or within the previous three years with any organizations that might have an interest in the submitted work. Other relationships: All authors have declared that there are no other relationships or activities that could appear to have influenced the submitted work.

\section{Acknowledgements}

The author would like to acknowledge Dr. M. Abass, Dr. I. Eljack, Dr. K. Salih, Dr. I. Jack, and my colleagues. Special thanks and appreciation to the college dean and administration of the College of Medicine, University of Bisha (Bisha, Saudi Arabia) for help and allowing the use of facilities.

\section{References}

1. Sekaran U, Bougie R: Research Methods For Business: A Skill Building Approach, 7th Ed. Sekaran U, Bougie R (ed): John Wiley \& Sons, Inc, Chichester; 2016

2. de Vaus DA: Research Design in Social Research. de Vaus DA (ed): Sage Publications, London; 2001.

3. Schoonenboom J, Johnson RB: How to construct a mixed methods research design. Kolner Z Soz Sozpsychol. 2017, 69:107-31. 10.1007/s11577-017-0454-1

4. Omair A: Selecting the appropriate study design for your research: descriptive study designs . J Health Spec. 2015, 3:153-56. 10.4103/1658-600X.159892

5. McKeon PO, Medina JM, Hertel J: Hierarchy of research design in evidence-based sports medicine . Athletic Therapy Today. 2006, 11:42-45.

6. Sayre JW, Toklu HZ, Ye F, Mazza J, Yale S: Case reports, case series - from clinical practice to evidencebased medicine in graduate medical education. Cureus. 2017, 9:e1546. 10.7759/cureus.1546

7. Parab S, Bhalerao S: Study designs. Int J Ayurveda Res. 2010, 1:128-31.

8. Baxter P, Jack S: Qualitative case study methodology: study design and implementation for novice researchers. Qualitative Report. 2008, 13:544-59.

9. Nissen T, Wynn R: The clinical case report: a review of its merits and limitations . BMC Res Notes. 2014, 7:264-71. 10.1186/1756-0500-7-264

10. Schneider D, Lilienfeld DE: Evidence-based Practices. Lilienfeld's Foundations of Epidemiology, 4th Edition. Schneider D, Lilienfeld DE (ed): Oxford University Press, New York; 2015. 285-96.

11. Chan K, Bhandari M: Three-minute critical appraisal of a case series article . Indian J Orthop. 2011, 45:103104. 10.4103/0019-5413.77126

12. Degen RM, Hodgins JL, Bhandari M: The language of evidence based medicine: answers to common questions?. Indian J Orthop. 2008, 42:111-17. 10.4103/0019-5413.40245

13. Hess DR: Retrospective studies and chart reviews. Respir Care. 2004, 49:1171-74.

14. Bhandari M, Joensson A: Clinical Research for Surgeons. Bhandari M, Joensson A (ed): Thieme, Stuttgart; 2011.

15. Lau F, Kuziemsky C: Handbook of eHealth Evaluation: An Evidence-based Approach (Internet). Lau F, Kuziemsky C (ed): University of Victoria, Victoria; 2016.

16. Gravetter FJ, Forzano LAB: Research Strategies and Validity. Research Methods for the Behavioral Sciences, 6th Edition. Gravetter FJ, Forzano LAB (ed): Wadsworth Publishing, Boston; 2018. 127-56.

17. Wang JJ, Attia J: Study designs in epidemiology and levels of evidence . Am J Ophthalmol. 2010, 149:367-70. 10.1016/j.ajo.2009.08.001

18. Grimes DA, Schulz KF: An overview of clinical research: the lay of the land . Lancet. 2002, 359:57-61. 10.1016/S0140-6736(02)07283-5

19. Setia MS: Methodology series module 3: cross-sectional studies . Indian J Dermatol. 2016, 61:261-64 10.4103/0019-5154.182410

20. Mann C: Observational research methods. Research design II: cohort, cross sectional, and case-control studies. Emerg Med J. 2003, 20:54-60. 10.1136/emj.20.1.54

21. Setia MS: Methodology series module 2: case-control studies . Indian J Dermatol. 2016, 61:146-51. 10.4103/0019-5154.177773

22. Song JW, Chung KC: Observational studies: cohort and case-control studies. Plast Reconstr Surg. 2010, 126:2234-42. 10.1097/PRS.0b013e3181f44abc

23. Eldredge J: Cohort studies in health sciences librarianship. J Med Libr Assoc. 2002 , 90:380-92. 


\section{Cureus}

24. Setia MS: Methodology series module 1: cohort studies . Indian J Dermatol. 2016, 61:21-25. 10.4103/00195154.174011

25. Prospective and Retrospective Cohort Studies. (2017). Accessed: December 27, 2019: http://sphweb.bumc.bu.edu/otlt/MPH-

Modules/EP/EP713_AnalyticOverview/EP713_AnalyticOverview3.html.

26. Gange SJ, Golub ET: Study Design. Infectious Disease Epidemiology: Theory and Practice, 3rd Edition. Nelson KE, Williams CM (ed): Jones \& Bartlett Learning, Burlington; 2014. 45-76.

27. Bickman L, Rog DL: Applied Research Design: A Practical Approach. The SAGE Handbook of Applied Social Research. Bickman L, Rog DL (ed): SAGE Publications, London; 2009. 3-46. 10.4135/9781483348858.n1 